\title{
PATRICK IGLESIAS
}

\section{Les $S O(3)$-variétés symplectiques et leur classification en dimension 4}

\author{
Bulletin de la S. M. F., tome 119, no 3 (1991), p. 371-396 \\ <http://www.numdam.org/item?id=BSMF_1991_119_3_371_0>
}

(C) Bulletin de la S. M. F., 1991, tous droits réservés.

L'accès aux archives de la revue «Bulletin de la S. M. F. » (http: //smf.emath.fr/Publications/Bulletin/Presentation.html) implique l'accord avec les conditions générales d'utilisation (http://www.numdam.org/ conditions). Toute utilisation commerciale ou impression systématique est constitutive d'une infraction pénale. Toute copie ou impression de ce fichier doit contenir la présente mention de copyright.

\section{NumDam}


Bull. Soc. math. France,

119, 1991, p. 371-396.

\title{
LES SO(3)-VARIÉTÉS SYMPLECTIQUES ET LEUR CLASSIFICATION EN DIMENSION 4
}

\author{
PAR \\ PATRICK IGLESIAS $(*)$
}

\begin{abstract}
RÉSumé. - On considère une variété symplectique $V$ munie d'une action symplectique et effective de $\mathrm{SO}(3)$. On montre que le stabilisateur principal de l'action $\mathrm{SO}(3)$ est du type $\mathrm{SO}(2)$ ou $\mathbb{Z} / m \mathbb{Z}$. Dans le premier cas, on montre que la variété $V$ est équivalente au produit symplectique d'une sphère $S^{2}$ par la variété des orbites $V / \mathrm{SO}(3)$. Le deuxième cas est traité uniquement en dimension 4 . On donne une classification complète, à isomorphisme près, des $\mathrm{SO}(3)$-variétés symplectiques de dimension 4 , compactes et non-compactes. Les seules $\mathrm{SO}(3)$-variétés symplectiques compactes de dimension 4 sont $S^{2} \times S^{2}$ et $P^{2}(\mathbb{C})$. La première peut être munie d'une infinité d'actions de $\mathrm{SO}(3)$ indexées par $\mathbb{N}$, la seconde est munie de l'action induite de celle, naturelle, de SU(3).

ABSTRACT. - We consider a symplectic manifold $V$ equipped with an effective and symplectic action of $\mathrm{SO}(3)$. We show that the principal stabilizer of the action of $\mathrm{SO}(3)$ has the type $\mathrm{SO}(2)$ or $\mathbb{Z} / m \mathbb{Z}$. In the first case, we show that $V$ is equivalent to the symplectic product of $S^{2}$ by the orbit manifold $V / \mathrm{SO}(3)$. The second case is treated only for $\operatorname{dim} V=4$. We give a complete classification, up to isomorphism, of the 4dimensional $\mathrm{SO}(3)$-symplectic manifolds, in the compact and non-compact case. The only compact $\mathrm{SO}(3)$-symplectic manifolds are $S^{2} \times S^{2}$ and $P^{2}(\mathbb{C})$. The first can be equipped with infinitely many different actions of $\mathrm{SO}(3)$ indexed by $\mathbb{N}$, the second is equipped with the natural action induced by $\mathrm{SU}(3)$.
\end{abstract}

\section{Introduction}

Cet article est une rédaction épurée des résultats obtenus en 1984 et présentés dans [Igl84], sur la classification des $\mathrm{SO}(3)$-variétés symplectiques de dimension 4.

Cette étude a été suggérée, à l'origine, par la physique : l'espace des mouvements $V$ d'un système dynamique est naturellement muni d'une forme symplectique, les symétries du système se traduisent par l'action

(*) Texte reçu le 18 décembre 1990.

P. Iglesias, Centre de Physique Théorique, case 907, LUMINY 13288 Marseille Cedex 9.

BULlETIN DE LA SOCIÉTÉ MATHÉMATIQUE DE FRANCE $\quad 0037-9484 / 1991 / 371 / \$ 5.00$

(C) Société mathématique de France 
symplectique ou hamiltonienne d'un groupe de Lie. D'après le principe de relativité galiléen, les systèmes dynamiques isolés ont le groupe de Galilée comme groupe de symétries. Les systèmes élémentaires - ceux pour lesquels l'action du groupe de Galilée est transitive - sont les revêtements des orbites coadjointes du groupe de Galilée [Sou70]. Dans le cas non transitif, lorsque le système est massif, c'est-à-dire quand une certaine classe de cohomologie est non-nulle, la variété des mouvements se décompose en un produit de deux variétés symplectiques, l'une représente les mouvements du centre de gravité et possède le groupe de Galilée comme groupe de symétries, l'autre est la variété des mouvements autour du centre de gravité et il ne lui reste de la symétrie galiléenne que l'action du groupe $\mathrm{SO}(3) \times \mathbb{R}$. Le premier cas non trivial est $\operatorname{dim} V=4$, c'est ce qui justifie cette étude.

On appelle $\mathrm{SO}(3)$-variété symplectique, toute variété symplectique $(V, \omega)$ connexe sans bord, muni d'une action différentiable symplectique effective de $\mathrm{SO}(3)$. Ces structures se partagent en 2 classes :

- le stabilisateur principal de $\mathrm{SO}(3)$ est de type $\mathrm{SO}(2)$;

- le stabilisateur principal de $\mathrm{SO}(3)$ est fini, de type $\mathbb{Z} / m \mathbb{Z}$.

On montre dans le premier cas, qu'en toute dimension, la variété $(V, \omega)$ est symplectomorphe au produit d'une variété symplectique $(X, \sigma)$ par une sphère symplectique $\left(S^{2}, a\right.$ Surf $)$ où Surf est le volume canonique de $S^{2}$ et $a$ un réel positif.

Le deuxième cas est plus compliqué, nous le traitons entièrement dans le cas $\operatorname{dim}(V)=4$ par des méthodes essentiellement topologiques. Nous donnons d'abord un tableau complet des variétés de dimension 4 compactes et non-compactes qui possèdent au moins une forme symplectique invariante par une action différentiable effective de $\mathrm{SO}(3)$. Ensuite nous classifions ces structures à difféomorphismes équivariants près. Nous introduisons pour cela une relation de pré-ordre naturelle sur les $\mathrm{SO}(3)$-variétés symplectiques dont nous classons seulement les éléments maximaux. Toute $\mathrm{SO}(3)$-variété symplectique de dimension 4 est alors symplectomorphe à un ouvert d'une de ces variétés maximales.

Nous montrons que les seules variétés compactes ainsi obtenues sont $S^{2} \times S^{2}$ qui possède une infinité dénombrable d'actions symplectiques non équivalentes de $\mathrm{SO}(3)$, et $P^{2}(\mathbb{C})$ qui n'en possède qu'une induite par l'inclusion $\mathrm{SO}(3) \subset \mathrm{SU}(3)$.

Certaines démonstrations originales (de [Igl84]) ont été modifiées et raccourcies et certaines constructions géométriques n'ont pas été incluses dans ce travail. Cet article aurait dû être publié en URSS où il a été achevé, mais pour une raison inconnue cela n'a pas été le cas, c'est ce qui explique sa publication tardive.

TOME $119-1991-\mathrm{N}^{\circ} 3$ 
Depuis la rédaction du travail original [Igl84], d'autres résultats relatifs à la classification de structures symplectiques hamiltoniennes globales ont été publiés, notamment par Thomas Delzant [Del88, Del90] et Michèle AUDIN [Aud88]. Le lecteur trouvera un exposé à peu près complet des résultats actuels sur la question des actions hamiltoniennes de groupe de Lie dans [Aud89].

Je remercie Michèle AUdin pour avoir lu et critiqué ce travail dans sa version originale, Thomas DELZANT pour ses remarques pertinentes, et François LAUDENBACH pour l'intérêt et l'aide qu'il m'a porté à sa réalisation dans cette nouvelle version.

\section{Actions symplectiques et hamiltoniennes}

Ce paragraphe est destiné à rappeler quelques constructions générales de géométrie symplectique que nous utiliserons dans la suite de cet article, $V$ désignera une variété connexe sans bord (compacte ou non), et $\omega$ une 2 -forme fermée : $\mathrm{d} \omega=0$, définie sur $V$. L'adjectif différentiable signifie indéfiniment différentiable.

Rappelons quelques termes de vocabulaire : $\omega$ est dite présymplectique si son feuilletage caractéristique $\operatorname{ker}(\omega)$ est de rang constant $>0$, symplectique s'il est nul $: \operatorname{ker}(\omega)=\{0\}$.

Considérons un groupe de Lie $G$ agissant différentiablement sur $V$, supposons que son action respecte la 2-forme fermée $\omega$ :

$$
\forall g \in G, \quad g^{*} \omega=\omega,
$$

notons $X_{u}$ le champs de vecteurs fondamental sur $V$ du vecteur $u \in \operatorname{Lie}(G)$ sur $V$, l'équation précédente (1) s'écrit infinitésimalement :

$$
\left.X_{u}(\omega)=0 \quad \Longrightarrow \quad \mathrm{d}\left(X_{u}\right\rfloor \omega\right)=0,
$$

où $X_{u}(\omega)$ désigne la dérivée de Lie de la forme $\omega$ par le champs $X_{u}$. On dit que l'action de $G$ est hamiltonienne pour $\omega$ si pour tout $u \in \operatorname{Lie}(G)$ la forme fermée $\left.X_{u}\right\rfloor \omega$ est exacte : il existe une fonction réelle $\psi_{u}$ telle que $\left.X_{u}\right\rfloor \omega=-\mathrm{d} \psi_{u}$. La fonction $\psi_{u}$ est un hamiltonien du champs $X_{u}$. On montre dans ces conditions qu'il existe une application, définie à une constante additive près, $\psi: V \rightarrow \operatorname{Lie}(G)^{*}$ telle que $\psi_{u}(x)=\psi(x) \cdot u$ [Sou70], en d'autres termes $\psi_{u}$ peut être choisie linéaire en $u$ :

$$
\left.\forall u \in \operatorname{Lie}(G), \quad \mathrm{d}\left(X_{u}\right\rfloor \omega\right)=-\mathrm{d} \psi \cdot u
$$

L'application $\psi$ est appelée le moment, relatif à $\omega$, de l'action de $G \operatorname{sur} V$. Remarquons qu'il n'est pas nécessaire que $\omega$ soit symplectique ni même 
présymplectique, il suffit qu'elle soit fermée. Si $F$ désigne une feuille du feuilletage caractéristique de $\omega$ et $\psi$ le moment de $G$, alors $\psi_{\mid F}$ est constante, c'est le théorème d'Emmy NæETHER.

Si $\omega$ est symplectique et $h$ est une fonction réelle différentiable, le champ de vecteurs défini par l'équation $\left.X_{h}\right\rfloor \omega=-\mathrm{d} h$ est appelé gradient symplectique de la fonction réelle $h$, il est noté aussi $\operatorname{grad} h$. Dans le cas de l'action du groupe $G, X_{u}$ est le gradient symplectique de la fonction réelle $\psi \cdot u$.

Remarque 2.1. - Il existe des conditions suffisantes à la fois sur la variété $V$ ou sur le groupe $G$ pour que toute action de $G \operatorname{sur} V$ qui préserve $\omega$ soit hamiltonienne, par exemple si la variété $V$ est simplement connexe, mais encore si l'algèbre de Lie du groupe est égale à son algèbre dérivée [Sou70], propriété vérifiée notamment par le groupe des rotations $\mathrm{SO}(3)$.

Le groupe $G$ agit sur le dual de son algèbre de Lie par l'action coadjointe, si $\mu \in \operatorname{Lie}(G)^{*}$ nous noterons $g \cdot \mu$ l'action coadjointe de $g \in G$ sur $\mu$. On montre que la fonction qui mesure le défaut d'équivariance du moment $\psi$, a priori définie sur $G \times V$ par $(g, x) \mapsto \psi(g \cdot x)-g \cdot \psi(x)$ ne dépend en fait que de $g$, on la note $\theta$, elle vérifie l'équation de cocycle : $\theta\left(g g^{\prime}\right)=g \cdot \theta\left(g^{\prime}\right)+\theta(g)$. Si $\theta$ est un cobord, c'est-à-dire s'il existe $\mu \in \operatorname{Lie}(G)^{*}$ tel que $\theta(g)=g \cdot \mu-\mu$, on peut ajouter $\mu$ au moment, ce qui le rend équivariant, on peut donc poser :

$$
\psi(g \cdot x)=g \cdot \psi(x)
$$

Remarque 2.2.. - La cohomologie de $G$ ne dépend pas de son action sur $(V, \omega)$, en particulier elle est nulle pour $\mathrm{SO}(3)$ : toute deux forme fermée $\omega$ sur $V$ invariante par $\mathrm{SO}(3)$ possède un moment équivariant.

Avant d'achever ce paragraphe signalons une formule que nous utiliserons sur la dimension du noyau de l'application moment, désignons par $\operatorname{Orth}_{\omega}(E)$ le sous-espace orthogonal symplectique au sous-espace vectoriel $E$ :

Proposition 2.3. - Soit $(V, \omega)$ une variété symplectique munie d'une action hamiltonienne d'un groupe de Lie $G$ de moment $\psi$. Désignons de façon générique par la lettre $\mathcal{O}$ l'orbite par $G$ des points de $V$, on a:

$$
\operatorname{ker}(\mathrm{d} \psi)=\operatorname{Orth}_{\omega}(T(\mathcal{O})) \Rightarrow \operatorname{dim}(\operatorname{ker}(\mathrm{d} \psi))=\operatorname{dim}(V)-\operatorname{dim}(\mathcal{O})
$$

ce qui implique encore $\operatorname{dim}(\operatorname{im}(\mathrm{d} \psi))=\operatorname{dim}(\mathcal{O})$.

TOMе $119-1991-\mathrm{N}^{\circ} 3$ 
Démonstration. - Soit $v \in \operatorname{ker}\left(\mathrm{d} \psi_{x}\right)$, pour tout $u \in \mathcal{G}$ algèbre de Lie de $G$, on a $\mathrm{d} \psi_{x}(v) \cdot u=0$, c'est-à-dire $\left.X_{u}\right\rfloor \omega_{x}(v)=0$, et comme $X_{u}(x)$ engendre $T_{x}(\mathcal{O})$ quand $u$ parcourt $\mathcal{G}$, on a la formule $\left.\operatorname{ker}(\mathrm{d} \psi)=\operatorname{Orth}_{\omega} T(\mathcal{O})\right)$. Pour démontrer le reste de la formule on utilise la propriété des formes symplectiques : $\operatorname{dim}(E)+\operatorname{dim}\left(\operatorname{Orth}_{\omega}(E)\right)=\operatorname{dim}(V)$, vrai pour tout sous-espace vectoriel (voir par exemple [Sou70]).

\section{Actions symplectiques de $\mathrm{SO}(3)$}

Dans tout ce qui suit on identifiera l'algèbre de Lie de $\mathrm{SO}(3)$ avec $\mathbb{R}^{3}$ grâce à l'opérateur produit vectoriel $j$ défini par : $j(x)(y)=x \wedge y$, et $\mathbb{R}^{3}$ avec son dual grâce au produit scalaire (forme de Killing). L'action coadjointe de $\mathrm{SO}(3)$ sur $\mathbb{R}^{3}$ s'identifiera alors à son action naturelle.

\subsection{Un hamiltonien particulier.}

On introduit ici un outil essentiel dans l'étude de l'action symplectique du groupe $\mathrm{SO}(3)$ : le hamiltonien canonique.

On considère une variété symplectique $(V, \omega)$ muni d'une action symplectique de $\mathrm{SO}(3)$. Compte tenu des remarques (2.1) et (2.2) on déduit que l'action de $\mathrm{SO}(3)$ sur $(V, \omega)$ est hamiltonienne et on note $L$ son moment équivariant. L'équation du moment (3) s'écrit, en notant $\langle\cdot \mid \cdot\rangle$ le produit scalaire usuel de $\mathbb{R}^{3}$ :

$$
\left.\forall u \in \mathbb{R}^{3}, \quad X_{u}\right\rfloor \omega=-\langle\mathrm{d} L \mid u\rangle
$$

L'équivariance du moment nous permet de construire le diagramme commutatif suivant :

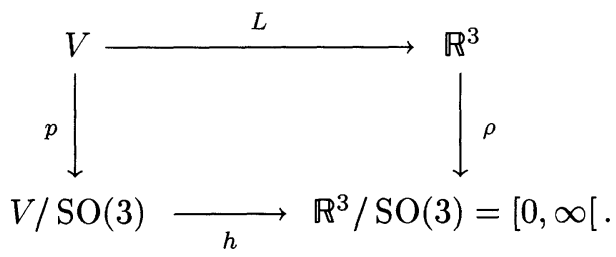

L'application $\rho$ défini par le diagramme ci-dessus est réalisée par le demi-carré $\rho(\xi)=\frac{1}{2}\|\xi\|^{2}$, on note :

$$
\mathcal{H}=\rho \circ L \quad \text { c'est-à-dire } \quad \mathcal{H}(x)=\frac{1}{2}\|L(x)\|^{2} .
$$


On appelle hamiltonien canonique et petit hamiltonien canonique les fonctions $\mathcal{H}$ et $h$. On déduit immédiatement de la formule (5) la formule :

$$
\operatorname{grad} \mathcal{H}(x)=X_{L(x)}(x)
$$

Le gradient du hamiltonien canonique $\mathcal{H}$ est complet (on dit que la fonction $\mathcal{H}$ est complète) et son exponentielle est donnée par l'action de l'exponentielle dans $\mathrm{SO}(3)$ de la matrice $j(L(x))$, c'est-à-dire :

$$
\mathrm{e}^{t \operatorname{grad} \mathcal{H}}(x)=\mathrm{e}^{t j(L(x))} x
$$

Il suffit de remarquer que le moment $L$ est invariant sous l'action du groupe à un paramètre qu'il engendre. On note de façon générale $e^{t f}$ la coulée engendrée par le champs de vecteurs $f$.

\subsection{Le type de stabilisateur principal.}

Nous renvoyons à BREDON [Bre72] et PALAIs [Pal60] pour la démonstration des théorèmes de la théorie des actions des groupes compacts sur les variétés que nous utilisons ici; nous y empruntons aussi le vocabulaire. Nous utiliserons notamment les termes d'orbite principale, orbite singulière, orbite singulière exceptionnelle (ce sont les orbites singulières de même dimension que les orbites principales), stabilisateur principal etc., rappelons que deux sous-groupes $H$ et $K$ d'un groupe $G$ ont même type s'ils sont conjugués.

La table des types de sous-groupes de $\mathrm{SO}(3)$ est donnée par :

1) il existe un seul sous-groupe de dimension 3, c'est $\mathrm{SO}(3)$ lui-même;

2) il existe deux types de sous-groupe de dimension 1 qui sont $\mathrm{SO}(2)$ et son normalisateur $\mathrm{O}(2)$;

3) les sous-groupes discrets sont les groupes cycliques à $m$ éléments $\mathbb{Z} / m \mathbb{Z}$, les groupes diédraux $\mathbb{D}_{2 m}=(\mathbb{Z} / 2 \mathbb{Z}) \rtimes(\mathbb{Z} / m \mathbb{Z})$ et les groupes $\mathbb{T}, \mathbb{O}$

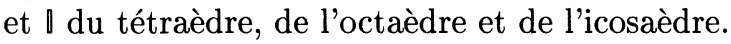

Nous renvoyons à WoLF [Wol72] pour la classification des sous-groupe discrets. De cette classification on déduit la nature du type de stabilisateur principal.

THÉORÈmE 3.1. - Le type de stabilisateur principal d'une action effective symplectique de $\mathrm{SO}(3)$ sur une variété symplectique $(V, \omega)$ est un sous-groupe de Lie de $\mathrm{SO}(2)$, c'est-à-dire : $\mathrm{SO}(2)$ lui-même ou $\mathbb{Z} / m \mathbb{Z}$, avec $m \in \mathbb{N}$.

Démonstration. - Soit $L$ le moment équivariant de l'action de $\mathrm{SO}(3)$ sur $V$, la condition d'équivariance $L(A \cdot x)=A L(x)$ implique l'inclusion

$$
\text { TOME } 119-1991-\mathrm{N}^{\circ} 3
$$


$\operatorname{St}(x) \subset \operatorname{St}(L(x))$. Or l'action coadjointe de $\mathrm{SO}(3)$ a deux types de stabilisateurs : $\mathrm{SO}(2)$ et $\mathrm{SO}(3)$. Compte tenu du tableau précédent des sous-groupes de $\mathrm{SO}(3)$, les seules possibilités d'inclusions sont données par :

$$
\left.\left.\begin{array}{l}
\mathrm{SO}(3) \\
\mathrm{O}(2) \\
\mathbb{D}_{2 m}, \mathbb{T}, \mathbb{O}, \mathbb{\Downarrow}
\end{array}\right\} \hookrightarrow \mathrm{SO}(3), \quad \begin{array}{l}
\mathrm{SO}(2) \\
\mathbb{Z} / m \mathbb{Z}
\end{array}\right\} \hookrightarrow \mathrm{SO}(2) \text { ou } \mathrm{SO}(3)
$$

Dans le premier cas le moment correspondant est nul, car $0 \in \mathbb{R}^{3}$ est le seul point qui a $\mathrm{SO}(3)$ pour stabilisateur, par densité le moment est nul partout : l'action de $\mathrm{SO}(3)$ est triviale par application de la formule du moment ce qui est exclu. Il ne reste donc que les cas annoncés.

\section{Cas de l'orbite principale $\mathbf{S}^{2}$}

Le cas du stabilisateur principal de type $\mathrm{SO}(2)$ se résoud complètement et ceci indépendamment de la dimension de la variété.

Soit $(V, \omega)$ une variété symplectique munie d'une action symplectique de $\mathrm{SO}(3)$. Nous supposerons que le type stabilisateur principal est $\mathrm{SO}(2)$. Nous allons montrer que $(V, \omega)$ est symplectomorphe au produit d'une variété symplectique $(M, \sigma)$ sur laquelle $\mathrm{SO}(3)$ agit trivialement par une sphère symplectique $\left(S^{2}, a \mathrm{Surf}\right)$ sur laquelle $\mathrm{SO}(3)$ agit naturellement, Surf est le volume canonique de $S^{2}$ et $a$ est un scalaire positif. Commençons par établir un certain nombre de propositions utiles.

Nous noterons : $V^{0}$ la réunion des orbites principales de $\mathrm{SO}(3), V^{*}$ la partie de $V$ sur laquelle le moment équivariant $L$ ne s'annulle pas, et enfin nous poserons $\widehat{V}=V^{0} \cap V^{*}$. La proposisition suivante est immédiate :

Proposition 4.1. - Compte tenu des notations précédentes, $\widehat{V}$ est un ouvert dense de $V$ saturé par l'action de $\mathrm{SO}(3)$.

Proposition 4.2. - Soient $(V, \omega)$ une variété symplectique munie d'une action symplectique de $\mathrm{SO}(3)$ et $L$ le moment équivariant. Si le type des orbites principales est $S^{2}$ alors $\operatorname{St}(x)=\operatorname{St}(L(x))$ pour tout $x \in V$, le hamiltonien canonique $\mathcal{H}$ est constant et toutes les orbites sont principales.

Démonstration. - Soit $x \in \widehat{V}$ (notations précédentes) le stabilisateur de $L(x)$ est le groupe des rotations autour de la doite $\mathbb{R} L(x)$, donc le type de $\operatorname{St}(L(x))$ est celui de $\operatorname{St}(x)$ or $\operatorname{St}(x) \subset \operatorname{St}(L(x))$ donc $\operatorname{St}(x)=$ $\operatorname{St}(L(x))$. Le gradient de $\mathcal{H}$ défini par l'action infinitésimale de $L$ est donc nul sur un ouvert dense de $V$, il est nul partout. On déduit que le 
hamiltonien canonique est constant sur $V$ ( $V$ est connexe), cette constante est nécessairement non nulle puisque l'action de $\mathrm{SO}(3)$ est effective. D'autre part $\mathrm{SO}(2)$ est conjugué à un sous-groupe de chaque stabilisateur non principal, ils ne peuvent donc être que de type $O(2)$ ou $\mathrm{SO}(3)$, mais le moment s'y annulerait ce qui n'est pas possible, donc tous les stabilisateurs sont de type $\mathrm{SO}(2)$ et sont conjugués entre eux (tous les sous-groupes de type $\mathrm{SO}(2)$ sont conjugués dans $\mathrm{SO}(3)$ ). On en déduit que toutes les orbites sont principales.

La variété $V$ est donc fibrée en sphères sur son quotient $V / \mathrm{SO}(3)$ qui est lui-même naturellement une variété, nous allons montrer, grâce à l'existence du moment équivariant $L$, que cette fibration est triviale.

THÉorÈme 4.3. - Soit $(V, \omega)$ une variété symplectique munie d'une action symplectique de $\mathrm{SO}(3)$. Si le type d'orbite principale est $S^{2}$ il existe sur la variété des orbites $Y=V / \mathrm{SO}(3)$ une structure symplectique $\sigma$ telle que $(V, \omega)$ soit symplectomorphe au produit de $(Y, \sigma)$ par une sphère symplectique ( $\left.S^{2}, a \mathrm{Surf}\right)$ où $\mathrm{SO}(3)$ agit naturellement sur $S^{2}$ et trivialement sur $Y$.

Démonstration. - En vertu de la Proposition 4.2 le moment équivariant $L$ est de module constant, on posera $\|L\|=a$. On peut donc définir l'application $\lambda=L / a$ à valeurs dans la sphère $S^{2}$. On choisit un point $u_{0} \in S^{2}$ et on pose $V_{0}=\lambda^{-1}\left(u_{0}\right)$, alors :

1) $V_{0}$ coupe toutes les orbites de $\mathrm{SO}(3)$ en un point et un seul : supposons le contraire, et soit $x$ et $x^{\prime}$ deux points de $V_{0}$ appartenant à une même orbite : $x^{\prime}=A x$ avec $A \in \mathrm{SO}(3)$, et $\lambda(x)=\lambda\left(x^{\prime}\right)=u_{0}$. Mais $\lambda$ est équivariant et donc $u_{0}=A u_{0}$, c'est-à-dire $A \in \operatorname{St}\left(u_{0}\right)$ or $\operatorname{St}(x)=\operatorname{St}\left(u_{0}\right)$ (Proposition 4.2) donc $A x=x$ c'est-à-dire $x=x^{\prime}$.

2) $V_{0}$ est une sous-variété de $V$ : on a montré (Proposition 2.3) que : $\operatorname{dim}\left(T_{x}(V)\right)=\operatorname{dim}\left(T_{x}(\mathcal{O})\right)+\operatorname{dim}\left(\operatorname{ker}\left(\mathrm{d} L_{x}\right)\right)$. On en déduit que $\mathrm{d} L_{x}$ est de rang constant et que $V_{0}$ est une sous-variété de dimension $\operatorname{dim}(V)-2$, nous l'identifierons au quotient $V / \mathrm{SO}(3)$.

3) Il existe un difféomorphisme équivariant de $V$ sur $Y \times S^{2}:$ soit $\pi$ la projection de $V$ sur $Y$, posons $\phi$ l'application définie sur $\mathrm{SO}(3) \times Y$ définie par $\phi(A, y)=A y, \phi$ est invariante par le sous-groupe des rotations autour $u_{0}$, elle se factorise donc en une application différentiable $\psi$ définie sur le produit $S^{2} \times Y$. L'inverse de $\psi$ est alors donnée par $\psi^{-1}(x)=$ $(\pi(x), \lambda(x))$ qui est évidemment différentiable. On déduit donc que $\psi$ est un difféomorphisme. Le transport de l'action de $\mathrm{SO}(3)$ est l'action annoncée : triviale sur $Y$ et naturelle sur $S^{2}$.

4) On transporte la structure symplectique de $V$ sur $Y \times S^{2}$, son moment équivariant s'écrit nécessairement $L(y, u)=\ell(y) u$ où $\ell$ est une

$$
\text { TOME } 119-1991-\mathrm{N}^{\circ} 3
$$


fonction réelle différentiable, or nous savons (4.2) que le hamiltonien canonique est une constante non nulle, donc $\ell(y)=a$ (à difféomorphisme près on peut choisir $a>0$ ). Considérons l'image réciproque sur $Y \times S^{2}$ de la forme symplectique $a$ Surf définie sur $S^{2}$, où Surf est le volume canonique, son moment équivariant est encore $L$, la différence avec la forme symplectique initiale est donc invariante par $\mathrm{SO}(3)$ et a dans son noyau les orbites de $\mathrm{SO}(3)$ : c'est l'image réciproque d'une 2-forme fermée de $Y$, notons la $\sigma$. On déduit que la forme symplectique s'écrit $a$ Surf $+\sigma$ (avec les abus de notation que cette écriture sous-entend). Il est immédiat ensuite de vérifier que $\sigma$ est régulière et donc qu'elle est symplectique. En fait $(Y, \sigma)$ s'identifie à la réduction symplectique à la Marsden-Weinstein [MW74] de $(V, \omega)$ à moment constant.

\section{Quelques propositions en dimension 4}

Nous donnons dans ce paragraphe quelques propositions utiles pour la classification ultérieure des $\mathrm{SO}(3)$-variétés symplectiques de dimension 4 . Rappelons que le cas intéressant est celui pour lequel le stabilisateur principal est cyclique de type $\mathbb{Z} / m \mathbb{Z}$ puisque la seule autre possibilité est $\mathrm{SO}(2)$ auquel cas la variété est un produit direct de la sphère par une autre variétéé symplectique ( $c f$. THÉORÈmES 3.1 et 4.3 ).

On considère l'application qui à toute 2-forme fermée $\omega$ invariante définie sur une $\mathrm{SO}(3)$-variété $V$ associe son moment équivariant $L$, nous allons montrer que cette application est injective, cette remarque ramène l'étude des structures symplectiques invariantes sur $V$ à celle d'une certaine partie de l'ensemble $\mathcal{E} \mathrm{q}\left(V, \mathbb{R}^{3}\right)$ des applications différentiables équivariantes de $V$ dans $\mathbb{R}^{3}$; nous caractériserons ensuite cet ensemble.

Proposition 5.1. - Soit $(V, \omega)$ une variété symplectique de dimension 4 munie d'une action symplectique de $\mathrm{SO}(3)$ à stabilisateur principal cyclique, alors $\omega$ est entièrement définie par son moment équivariant $L$. En d'autres termes si $\omega^{\prime}$ est une autre forme symplectique sur $V$ invariante par $\mathrm{SO}(3)$ et de même moment équivariant $L$, elle est égale à $\omega$.

Démonstration. - Soit $\omega$ et $\omega^{\prime}$ un couple de 2-formes fermées sur $V$ invariantes par $\mathrm{SO}(3)$ et possédant un même moment équivariant $L$. Leur différence $\alpha=\omega-\omega^{\prime}$ est une 2-forme invariante par $\mathrm{SO}(3)$ qui a les orbites de $\mathrm{SO}(3)$ dans son noyau; en effet l'équation du moment permet d'écrire $\left.X_{u}\right\rfloor \alpha=0$ pour tout $u \in \mathbb{R}^{3}$. Ainsi $\alpha$ est l'image réciproque d'une 2-forme sur l'espace des orbites $V / \mathrm{SO}(3)$ qui est de dimension 1 et donc $\alpha$ est nulle, c'est-à-dire $\omega=\omega^{\prime}$. 
Remarque 5.2. - Il est clair que la proposition précédente s'applique encore quand on considère l'action d'un groupe de Lie dont les orbites principales sont de codimension 1 ; l'ensemble des structures symplectiques est encore un sous-ensemble de $\mathcal{E} \mathrm{q}\left(V, \mathcal{G}^{*}\right)$.

Proposition 5.3. - Soit $(V, \omega)$ une $\mathrm{SO}(3)$-variété symplectique de dimension 4 : ou bien le type de stabilisateur principal est $\mathrm{SO}(2)-e t$ alors $V$ est isomorphe au produit de $S^{2}$ par une variété symplectique $W$ (théorème 4.3) - ou bien le type de stabilisateur principal est cyclique de type $\mathbb{Z} / m \mathbb{Z}$ et toute orbite singulière est du type $S^{2}$ ou $P^{2}(\mathbb{R})$ : il n'y a pas d'orbite singulière exceptionnelle.

Démonstration. - On suppose donc que le type de stabilisateur principal est cyclique, et soit $x \in V$ un point dont l'orbite $\mathcal{O}$ est de dimension 3 ; grâce à l'équation du moment on obtient $: \operatorname{ker}\left(\mathrm{d}\|L\|^{2}\right)=$ $\operatorname{Orth}_{\omega}\left(X_{L(x)}(x)\right)$; le stabilisateur de $x$ étant discret, on en déduit que $\operatorname{dim} \operatorname{ker}\left(\mathrm{d}\|L\|^{2}\right)=3$; or si $x$ est sur une orbite singulière : $\mathrm{d}\|L\|^{2}=0$ c'est-à-dire dim $\operatorname{ker}\left(\mathrm{d}\|L\|^{2}\right)=4$; donc $x$ est principal.

Proposition 5.4. - Soit $V$ une variété de dimension 4 munie d'une action différentiable effective de $\mathrm{SO}(3)$ à type de stabilisateur principal cyclique. Soit $\omega$ une 2 -forme fermée sur $V$ invariante par $\mathrm{SO}(3)$ de moment équivariant $L, \omega$ est symplectique si et seulement si son petit hamiltonien canonique $h$, défini sur $V / \mathrm{SO}(3)$ à valeurs dans $\mathbb{R}$ (diagramme 6) est un plongement propre. En d'autres termes, $h$ est un difféomorphisme de $V / \mathrm{SO}(3)$ sur son image $h(V / \mathrm{SO}(3)) \subset \mathbb{R}$. En particulier $V / \mathrm{SO}(3)$ ne peut pas être du type $S^{1}$.

Démonstration. - Soit $x$ un point de $V, \mathcal{O}$ l'orbite de $x$ et $\mathcal{H}$ le hamiltonien canonique de $\omega$ (formule (7)).

1) Supposons que $\mathcal{O}$ soit une orbite principale, si $\omega$ est régulière alors $\mathrm{d} \mathcal{H}$ est de rang 1 , son noyau est $T_{x}(\mathcal{O})$. En effet, remarquons d'abord que $L(x)$ ne s'annulle pas (sinon l'orbite $\mathcal{O}$ serait isotrope et ne pourrait pas être de dimension 3) et $\operatorname{rg}\left(\mathrm{d} L_{x}\right)=\operatorname{dim}(\mathcal{O})=3$ (Proposition 2.3). Donc $\mathrm{d} \mathcal{H}_{x}=0 \Rightarrow L(x) \perp \operatorname{im}\left(\mathrm{d} L_{x}\right)$ (formule du moment) et alors $L(x)=0$ ou $\operatorname{rg}\left(\mathrm{d} L_{x}\right)<3$, ce qui est impossible. Réciproquement supposons que $x$ soit sur une orbite principale et $\operatorname{ker}\left(\mathrm{d} \mathcal{H}_{x}\right)=T_{x}(\mathcal{O})$, on décompose $T_{x}(V)$ en la somme de $T_{x}(\mathcal{O})$ et d'un espace vectoriel $E$ transverse au précédent. Tout vecteur $\delta x \in T_{x}(V)$ est la somme d'un vecteur $X_{u}(x), u \in \mathbb{R}^{3}$, et d'un élément $v \in E$ vérifiant $L(x) \cdot \mathrm{d} L_{x}(v)=0$ si et seulement si $v=0$. Soit alors $\delta x=X_{u}(x)+v$ et $\delta^{\prime} x=X_{u^{\prime}}(x)+v^{\prime}$ deux vecteurs de $T_{x}(V)$, et écrivons $\omega_{x}\left(\delta x, \delta^{\prime} x\right)=0$ pour tout $\delta^{\prime} x$. Ceci s'écrit encore :

$$
u \wedge L(x)+\mathrm{d} L_{x}(v)=0 \quad \text { et } \quad u \cdot \mathrm{d} L_{x}=0
$$

TOME $119-1991-\mathrm{N}^{\circ} 3$ 
en multipliant par $L(x)$ la première équation on obtient $v \in \operatorname{ker}\left(\mathrm{d}\|L\|_{x}^{2}\right)$ ce qui implique $v=0$, il reste alors $u=\lambda L(x)$ avec $\lambda \in \mathbb{R}$. Reporté dans la deuxième équation on obtient $\lambda \mathrm{d}\|L\|_{x}^{2}=0$ ce qui implique $\lambda=0$. Ainsi $\delta x=0$ et $\omega$ est régulière. Nous voyons déjà que $V / \operatorname{SO}(3) \neq S^{1}$.

2) Considérerons maintenant le cas où $\mathcal{O}$ est une orbite singulière, et soit $H$ le stabilisateur de $x$; en fait $H$ est $\mathrm{SO}(2)$ ou $\mathrm{O}(2)$ et on ne perd pas en généralité à supposer que $H=\mathrm{SO}(2)$ car seule son action infinitésimale va intervenir ici. D'après le théorème de la tranche [Bre72] on sait que l'orbite singulière $\mathcal{O}$ de $x$ possède un voisinage équivariant $\mathcal{U}$ isomorphe au fibré associé $\mathrm{SO}(3) \times_{H} \mathbb{R}^{2}$, où $\mathrm{SO}(2)$ agit orthogonalement sur $\mathbb{R}^{2}$ et transitivement sur la sphère unité. La projection $p_{\mid \mathcal{U}}$ de $\mathcal{U}$ sur $\mathcal{U} / \mathrm{SO}(3)$ se relève sur $\mathrm{SO}(3) \times \mathbb{R}^{2}$ par une fonction (différentiable) du module carré $\|\xi\|^{2}$ où $\xi$ est la variable de $\mathbb{R}^{2}$. Nous noterons $\tilde{\omega}$ le relevé de $\omega_{\mid \mathcal{u}}$ sur $\mathrm{SO}(3) \times \mathbb{R}^{2}$, et $\tilde{L}$ le relevé de $L$ sur $\mathrm{SO}(3) \times \mathbb{R}^{2} ;$ par raison d'équivariance : $\tilde{L}(g, \xi)=g \ell(\xi)$ où $\ell \in \mathcal{C}^{\infty}\left(\mathbb{R}^{2}, \mathbb{R}^{3}\right)$. La dérivée extérieure de la 1 -forme $\alpha$ définie avec quelques abus de notations par $\alpha=\ell \cdot \theta$ (où le point désigne le produit scalaire ordinaire dans $\mathbb{R}^{3}$ et $\theta$ la forme de MaurerCartan de $\mathrm{SO}(3)$ ) est une 2-forme fermée invariante par $\mathrm{SO}(3)$ ayant $\tilde{L}$ comme moment équivariant; la différence avec $\tilde{\omega}$ est l'image réciproque d'une 2-forme fermée $\sigma$ définie sur $\mathbb{R}^{2}$, nous pouvons donc écrire avec quelques abus :

$$
\tilde{\omega}=\mathrm{d}[\ell . \theta]+a \operatorname{Surf}_{\mathbb{R}^{2}}
$$

où $a$, en raison de l'invariance de $\tilde{\omega}$ par $\mathrm{SO}(2)$ (l'action de $\mathrm{SO}(3)$ est effective), est une fonction différentiable de $\|\xi\|^{2}$. La condition du moment $\tilde{L}$ par $\mathrm{SO}(2)$ s'écrit :

$$
\ell\left(\gamma^{m} \xi\right)=\gamma \ell(\xi) \quad \forall \gamma \in \mathrm{SO}(2)
$$

Les seules actions orthogonales de $\mathrm{SO}(2)$ sur $\mathbb{R}^{2}$ transitives sur la sphère unité s'écrivent $(\gamma, \xi) \mapsto \gamma^{m} \xi$. Le forme $\tilde{\omega}$ est présymplectique; elle a comme noyau l'action infinitésimale de $\mathrm{SO}(2)$, le noyau de $\tilde{\omega}$ est donc dirigé par le vecteur $X(g, \xi)=(-g j(k), m k \wedge \xi)$; c'est cette condition que nous allons exploiter maintenant. Pour la commodité du calcul nous avons identifié $\mathbb{R}^{2}$ avec le plan orthogonal au troisième vecteur de base $k=(0,0,1) \in \mathbb{R}^{3}$ et $\mathrm{SO}(2)$ avec les rotations autour de $k$, après quelques manipulations le calcul donne :

$$
X \in \operatorname{ker}(\tilde{\omega}) \Rightarrow a(\rho)=\frac{1}{2 m \rho}\left\langle\mathrm{d} \ell_{\xi}(\xi) \mid k\right\rangle \quad \rho=\frac{1}{2}\|\xi\|^{2}
$$


La condition donnée par l'équation précédente (11) implique que $\ell(0)$ soit parallèle au vecteur $k$, ce qui nous permet de décomposer $\ell$ de la façon suivante :

$$
\ell(\xi)=p(\rho) k+q(\rho) \xi+r(\rho) k \wedge \xi .
$$

C'est par raison d'équivariance que les fonctions $p, q$ et $r$ ne dépendent de $\xi$ que par $\rho$; on peut montrer qu'elles sont différentiables dans cette variable (certaines sont nulles pour $m>1$, nous le verrons en détail plus loin). La condition sur la fonction $a$ (équation (12)) permet d'écrire alors :

$$
\tilde{\omega}=\mathrm{d}[\ell \cdot \theta]+\frac{p^{\prime}}{m} \operatorname{Surf}_{\mathbb{R}^{2}} .
$$

Nous savons que hors de l'orbite singulière la condition pour $\omega$ d'être symplectique est que son petit hamiltonien canonique $h$ n'ait pas de point critique; ce qui nous intéresse donc ici est le comportement de $\omega$ sur l'orbite singulière dont la préimage sur $\mathrm{SO}(3) \times \mathbb{R}^{2}$ est définie par l'équation $\xi=0$. Il est nécessaire que le noyau de $\tilde{\omega}$ soit réduit à l'action infinitésimale de $\mathrm{SO}(2)$. Puisque $\tilde{\omega}$ est invariante par $\mathrm{SO}(3)$, il est suffisant de calculer son noyau au point de l'orbite singulière $(\mathbf{1}, 0)$, en utilisant l'identification de l'algèbre de Lie de $\mathrm{SO}(3)$ avec $\mathbb{R}^{3}$ on peut écrire :

$$
\begin{aligned}
(u, v) \in \operatorname{ker}\left(\tilde{\omega}_{(\mathbf{1}, 0)}\right) \subset T_{(\mathbf{1}, 0)}\left(\mathrm{SO}(3) \times \mathbb{R}^{2}\right) \\
\Longrightarrow\left\{\begin{array}{l}
\ell^{\prime}(0)(v)+u \wedge \ell(0)=0, \\
p^{\prime}(0) k \wedge^{t} \mathrm{~d} \ell(u)=\lambda k,
\end{array}\right.
\end{aligned}
$$

où $\lambda$ est un multiplicateur de Lagrange lié à la condition $\langle\xi \mid k\rangle=0$. En décomposant $v$ sur $k$ et $\mathbb{R}^{2}: v=v_{k} k+\nu$ on obtient le système à quatre inconnues

$$
(u, v) \in \operatorname{ker}\left(\tilde{\omega}_{(\mathbf{1}, 0)} \Rightarrow M\left(\begin{array}{l}
u \\
v
\end{array}\right)=0\right.
$$

avec :

$$
M=\left(\begin{array}{cc}
p^{\prime}(0) j(k) & -q(0) \mathbf{1}+r(0) j(k) \\
q(0) \mathbf{1}+r(0) j(k) & -p(0) j(k)
\end{array}\right) .
$$

Pour que $\operatorname{ker}(\tilde{\omega})$ soit réduit à l'espace tangent à l'orbite de $\operatorname{SO}(2)$ il faut et il suffit que cette matrice $M$ soit régulière. En remarquant que $j(k)$ est, dans le plan $\mathbb{R}^{2}$, la rotation d'angle $\frac{1}{2} \pi$, le calcul du déterminant donne :

$$
\left[p(0) p^{\prime}(0)+q(0)^{2}+r(0)^{2}\right] \neq 0 \Rightarrow h^{\prime}(0) \neq 0,
$$


où $h$ est exprimé dans la variable $\rho$. La condition de régularité de $\omega$ implique donc que le petit hamiltonien canonique n'a pas de point critique sur l'espace des orbites, c'est-à-dire que $h: V / \mathrm{SO}(3) \rightarrow \mathbb{R}^{3} / \mathrm{SO}(3)$ est un plongement propre.

\section{Modèles des $\mathrm{SO}(3)$-variétés symplectiques de dimension 4}

Dans ce paragraphe nous donnons une liste de $\mathrm{SO}(3)$-variétés compactes ou non, munies chacune d'une famille de formes symplectiques. Nous ne donnons pas tous les détails des calculs et les expressions des formes symplectiques, le lecteur intéressé pourra se reporter à [Igl84]. Nous montrerons ensuite que toute $\mathrm{SO}(3)$-variété symplectique de dimension 4 est symplectomorphe à un de ces modèles.

Nous utilisons tout le long sans le dire le théorème suivant sur les singularités d'applications $\mathcal{C}^{\infty}$ en présence de symétries [Poé76] : l'ensemble des applications différentiables équivariantes d'une $G$-variété ( $G$ compact) $X$ vers un $G$-espace vectoriel $E$ est un module de type fini sur l'algèbre des fonctions réelles invariantes définies sur $X$.

\subsection{Les variétés $\mathcal{L}_{m}$.}

Considérons la $\mathrm{SO}(3)$-variété $\mathcal{L}_{1}=\mathrm{SO}(3) \times \mathbb{R}$ où $\mathrm{SO}(3)$ agit naturellement sur $\mathrm{SO}(3)$ et trivialement sur $\mathbb{R}$. Toute fonction équivariante $L \in \mathcal{E} \mathrm{q}\left(\mathcal{L}_{1}, \mathbb{R}^{3}\right)$ s'écrit :

$$
L(g, t)=g \ell(t) \quad \text { avec } \quad \ell \in \mathcal{C}^{\infty}\left(\mathbb{R}, \mathbb{R}^{3}\right) .
$$

En vertu de la Proposition 5.4, $L$ est le moment d'une forme symplectique invariante sur $\mathcal{L}_{1}$ si et seulement si son petit hamiltonien canonique est sans points critiques, c'est-à-dire si :

$$
h^{\prime}(t) \neq 0 \quad \forall t \in \mathbb{R} \quad \text { avec } \quad h=\frac{1}{2}\|\ell\|^{2} .
$$

La dérivée extérieure $\omega$ de la forme $\ell \cdot \theta$, où $\theta$ est la forme de Maurer-Cartan de $\mathrm{SO}(3)$ et le point désigne le produit scalaire de $\mathbb{R}^{3}$, a évidemment $L$ comme moment; en vertu de la Proposition 5.1, c'est la forme recherchée, on peut donc écrire :

$$
\omega=\mathrm{d}[\ell \cdot \theta]=\mathrm{d} \ell \wedge \theta+\ell \cdot \mathrm{d} \theta .
$$

Considérons maintenant les variétés :

$$
\mathcal{L}_{m}=(\mathrm{SO}(3) /[\mathbb{Z} / m \mathbb{Z}]) \times \mathbb{R} \text { avec } m \in \mathbb{N}-\{0\} .
$$


Elles sont le quotient de $\mathcal{L}_{1}$ par $\mathbb{Z} / m \mathbb{Z}$ qui en est un revêtement. Toute forme symplectique invariante définie sur $\mathcal{L}_{m}$ se relève sur $\mathcal{L}_{1}$ en une forme symplectique invariante par $\mathrm{SO}(3)$ et par $\mathbb{Z} / m \mathbb{Z}$ agissant à droite. Le moment $\tilde{L}$ de $\mathrm{SO}(3)$ relevé sur $\mathcal{L}_{1}$ est donc une fonction équivariante par $\mathrm{SO}(3)$ et invariante par $\mathbb{Z} / m \mathbb{Z}$. On a donc $\tilde{L}(g, t)=g \tilde{\ell}(t)$ avec $a \ell=\ell$ pour tout $a \in \mathbb{Z} / m \mathbb{Z}$. Pour $m>1$, en réalisant $\mathbb{Z} / m \mathbb{Z}$ comme un sousgroupe des rotations autour de l'axe dirigé par le vecteur $k=(0,0,1)$, ceci implique : $\tilde{\ell}$ parallèle à $k$, et donc :

$$
\tilde{\ell}(t)=\lambda(t) k \quad \text { avec } \quad \lambda \in \mathcal{C}^{\infty}(\mathbb{R}, \mathbb{R}) .
$$

La condition de la Proposition 5.4 s'écrit alors :

$$
\forall t \in \mathbb{R}, \quad \lambda(t) \neq 0 \text { et } \lambda^{\prime}(t) \neq 0
$$

L'analyse en type d'orbites de l'action de $\mathrm{SO}(3)$ sur $\mathcal{L}_{m}$ est simple puisque toutes les orbites sont principales, les stabilisateurs sont tous du type $\mathbb{Z} / m \mathbb{Z}$.

\subsection{Les fibrés $\mathcal{F}_{m}$.}

On considère la fibration naturelle $\pi: \mathrm{SO}(3) \rightarrow S^{2}$, pour tout entier $m>0$ on fait agir $\mathrm{SO}(2)$ sur $\mathbb{R}^{2}$ par :

$$
\forall a \in \mathrm{SO}(2), \quad \forall \xi \in \mathbb{R}^{2}, \quad(a, \xi) \mapsto a^{m} \xi .
$$

On note $\mathcal{F}_{m}=\mathrm{SO}(3) \times_{\mathrm{SO}(2)} \mathbb{R}^{2}$ le fibré associé à $\pi$ par cette action et :

$$
\operatorname{pr}_{m}: \mathcal{F}_{m} \rightarrow S^{2}
$$

la projection canonique sur sa base. Soit $f_{m}: S^{2} \rightarrow S^{2}$ l'application de degré $m$ qui s'écrit en coordonnées projectives :

$$
f_{m}: z \mapsto z^{m} .
$$

Le fibré $\mathcal{F}_{m}$ est alors isomorphe à $f_{m}^{*}\left(\mathcal{F}_{1}\right)$ et $\mathcal{F}_{1}$ s'identifie naturellement au fibré tangent $T S^{2}, 2 m$ est la classe de Chern de $\mathcal{F}_{m}$. On peut écrire :

$$
\mathcal{F}_{m} \simeq f_{m}^{*}\left(T S^{2}\right)=\left\{(x, r) \in S^{2} \times \mathbb{R}^{3} ;\left\langle f_{m}(x) \mid r\right\rangle=0\right\} .
$$

Comme on peut le constater, cette formule (28) permet aussi de construire un plongement canonique de $\mathbb{Z} / m \mathbb{Z}$ dans $S^{2} \times S^{2}$ par :

$$
\mathbb{Z} / m \mathbb{Z} \simeq\left\{(x, y) \in S^{2} \times S^{2} ;\left\langle f_{m}(x) \mid y\right\rangle=0\right\} .
$$

TOME $119-1991-\mathrm{N}^{\circ} 3$ 
Il existe alors un cocycle différentiable $\psi_{m}: \mathrm{SO}(3) \times S^{2} \rightarrow \mathrm{SO}(3)$ vérifiant les propriétés suivantes [Igl84] :

$$
\forall A, B \in \mathrm{SO}(3), \forall x \in S^{2}, \quad\left\{\begin{array}{l}
\psi_{m}(A B, x)=\psi_{m}(A, B x) \psi_{m}(B, x) \\
f_{m}(A x)=\psi_{m}(A, x) f_{m}(x) .
\end{array}\right.
$$

Ce qui permet de construire l'action suivante de $\operatorname{SO}(3) \operatorname{sur} \mathcal{F}_{m}$ :

$$
\forall A \in \mathrm{SO}(3), \forall(x, r) \in \mathcal{F}_{m}, \quad(A, x, r) \mapsto\left(A x, \psi_{m}(A, x) r\right) .
$$

On constate que pour $m=1$ cette action redonne l'action canonique sur $T S^{2}$.

L'espace des orbites $\mathcal{F}_{m} / \mathrm{SO}(3)$ s'identifie avec la demi-droite $[0, \infty[$ grâce à la projection :

$$
\forall(x, r) \in \mathcal{F}_{m}, \quad(x, r) \mapsto \rho=\frac{1}{2}\|r\|^{2} .
$$

Toute application équivariante $L \in \mathcal{E} \mathrm{q}\left(\mathcal{F}_{m}, \mathbb{R}^{3}\right)$ s'écrit alors :

$$
\forall(x, r) \in \mathcal{F}_{m}, \quad L(x, r)=a(\rho) x+b(\rho) r+c(\rho) x \wedge r,
$$

où $a, b, c$ sont des fonctions différentiables sur la demi-droite $[0, \infty[$. Pour $m>1$ les fonctions $b$ et $c$ sont identiquement nulles. Chaque application équivariante $L$ définie par l'équation (33) est le moment d'une 2-forme fermée invariante que nous noterons $\sigma_{m}$ et dont nous allons donner l'expression. Considérons pour cela la 1 -forme $\gamma_{m}$ définie sur $S^{2}$ par la formule suivante :

$$
\operatorname{Hopf}^{*}\left(\gamma_{m}\right)=\left[1-\frac{1}{2} \phi_{m}\right]^{*}(\omega),
$$

où Hopf : $S^{3} \rightarrow S^{2}$ est, comme son nom l'indique, la fibration de Hopf et $\omega$ la forme de connexion canonique pour l'action de $U(1)$. L'application $\phi_{m}: S^{3} \rightarrow S^{3}$ relève de façon canonique l'application $f_{m}$ en une application de degré $m$ :

$$
\text { Hopf } \circ \phi_{m}=f_{m} \circ \text { Hopf, }
$$

le lecteur qui le désire pourra trouver les détails de cette construction dans [Igl84]. Compte tenu des notations introduites par la formule (33), posons :

$$
\begin{array}{r}
w_{m}=\frac{a(\rho)-a(0)}{2 m \rho}\left\langle f_{m}(x) \mid r \wedge \mathrm{d} r\right\rangle+b(\rho)\left\langle r \mid f_{m}(x) \wedge \mathrm{d} x\right\rangle \\
+c(\rho)\langle r \mid \mathrm{d} x\rangle+[a(\rho)-a(0)] \gamma_{m} .
\end{array}
$$

BULLETIN DE LA SOCIÉTÉ MATHÉMATIQUE DE FRANCE 
Rappelons que pour $m>1$ les fonctions $b$ et $c$ sont nulles et que pour $m=1$ c'est la 1 -forme $\gamma_{1}$ qui est nulle. La 2-forme $\sigma_{m}$ définie sur $\mathcal{F}_{m}$ ayant $L$ (formule (33)) pour moment s'écrit alors :

$$
\sigma_{m}=\mathrm{d} w_{m}-a(0) \operatorname{pr}_{m}^{*}(\text { Surf }) .
$$

La 2-forme Surf est l'élément de volume canonique de la sphère $S^{2}$. Pour que $\sigma_{m}$ soit symplectique il faut et il suffit que le petit hamiltonien canonique :

$$
h: \rho \mapsto a(\rho)^{2}+2 \rho\left[b(\rho)^{2}+c(\rho)^{2}\right]
$$

soit sans points critiques sur la demi-droite $[0, \infty[$ (Proposition 5.4). Remarquons que la valeur $a(0)$ représente la classe de cohomologie de $\sigma_{m}$. Elle n'est jamais nulle si $m>1$, le caractère non critique de $h$ au point $\rho=0$ l'interdit.

L'analyse en type d'orbites de l'action de $\mathrm{SO}(3)$ sur $\mathcal{F}_{m}$ est immédiate : il y a une orbite singulière de type $S^{2}$, c'est la section nulle de la fibration $\operatorname{pr}_{m}: \mathcal{F}_{m} \rightarrow S^{2}$; les autres orbites sont principales, les stabilisateurs principaux sont du type $\mathbb{Z} / m \mathbb{Z}$.

6.3. Le tangent à $P^{2}(\mathbb{R})$.

Le groupe $\mathrm{SO}(3)$ agit naturellement sur l'espace tangent au projectif réel $P^{2}(\mathbb{R})$ dont le revêtement universel est $T S^{2}$, l'action de $\mathrm{SO}(3)$ sur $P^{2}(\mathbb{R})$ relevée à $T S^{2}$ est évidemment son action naturelle. Toute forme symplectique sur $T P^{2}(\mathbb{R})$ invariante par $\mathrm{SO}(3)$ se relève donc en une forme symplectique sur $T S^{2}$ invariante par $\mathrm{SO}(3)$ et par $\mathbb{Z} / 2 \mathbb{Z}$. Cette remarque nous permet d'identifier les structures symplectiques invariantes sur $T P^{2}(\mathbb{R})$ avec une famille de structures symplectiques invariantes sur $T S^{2}$. Le moment équivariant relevé sur $T S^{2}$ est lui invariant par $\mathbb{Z} / 2 \mathbb{Z}$ dont l'élément non trivial -1 agit par :

$$
\forall(x, r) \in T S^{2}, \quad-1:(x, r) \mapsto(-x,-r) .
$$

En considérant les notations du paragraphe précédent (formule (33)) on déduit :

$$
L(x, r)=c(\rho) x \wedge r .
$$

La forme symplectique $\sigma$ dont $L$ est le moment est alors donné par la formule :

$$
\sigma=\mathrm{d} w \quad \text { avec } \quad w=c(\rho)\langle r \mid \mathrm{d} x\rangle
$$

TOME $119-1991-\mathrm{N}^{\circ} 3$ 
Remarque 6.1. - Sur $T P^{2}(\mathbb{R})$, la classe de cohomologie de $\sigma$ est toujours nulle. L'analyse en type d'orbites de l'action de $\mathrm{SO}(3) \operatorname{sur} T P^{2}(\mathbb{R})$ nous donne une orbite singulière de type $P^{2}(\mathbb{R})$, c'est la section nulle; les autres orbites sont principales les stabilisateurs sont du type $\mathbb{Z} / 2 \mathbb{Z}$. La SO(3)-variété $T P^{2}(\mathbb{R})$ peut encore être obtenu comme le produit croisé $\mathrm{SO}(3) \times \times_{\mathrm{O}(2)} \mathbb{R}^{2}$ où $\mathrm{O}(2)$ naturellement sur $\mathrm{SO}(3)$ et $\mathbb{R}^{2}$.

\subsection{Les variétés $\mathcal{S}_{m}$.}

Considérons à nouveau la famille de cocycles $\psi_{m}$ définis en (30); elle nous permet de définir pour chaque entier $m \geq 1$ une action différente de $\mathrm{SO}(3)$ sur le produit direct $S^{2} \times S^{2}$ :

$$
\forall A \in \mathrm{SO}(3), \forall(x, y) \in S^{2} \times S^{2}, \quad(A, x, y) \mapsto\left(A x, \psi_{m}(A, x) y\right)
$$

Nous noterons $\mathcal{S}_{m}$ la $\mathrm{SO}(3)$-variété $S^{2} \times S^{2}$ munie de cette action. La variété $\mathcal{S}_{1}$ n'est autre que $S^{2} \times S^{2}$ munie de l'action diagonale. L'espace des orbites $\mathcal{S}_{m} / \mathrm{SO}(3)$ est réalisée par le segment $[-1,+1]$ grâce à la projection :

$$
\forall(x, y) \in S^{2} \times S^{2}, \quad(x, y) \mapsto \rho=\left\langle f_{m}(x) \mid y\right\rangle,
$$

où la fonction $f_{m}$ est définie dans le $\S 6.2$. Toute fonction équivariante $L \in \mathcal{E} \mathrm{q}\left(\mathcal{S}_{m}, \mathbb{R}^{3}\right)$ s'écrit :

$$
L(x, y)=a(\rho) x+b(\rho) y+c(\rho) x \wedge y,
$$

où $a, b$ et $c$ sont des fonctions différentiables de $[-1,+1]$ dans $\mathbb{R}$. Pour $m>1$ les fonctions $b$ et $c$ sont identiquement nulles. Chaque fonction équivariantes $L$ est le moment d'une 2-forme $\sigma_{m}$. Nous donnons l'expression de $\sigma_{1}$ à titre de curiosité et laissons le soin au lecteur, s'il le désire, de calculer l'expresion de $\sigma_{m}$ en toute généralité. Indiquons simplement qu'il suffit de plonger de façon équivariante le fibré $\mathcal{F}_{m}$ dans $\mathcal{S}_{m}$, qui s'identifie à un voisinage tubulaire de chaque orbite singulière de l'action de $\mathrm{SO}(3)$ (voir [Igl84]), et l'expression générale utilise la forme $\gamma_{m}$ introduite au $\S 6.2$.

$$
\begin{aligned}
\sigma_{1}=(\rho a+b)^{\prime} \operatorname{Surf}_{1}+(\rho b+a)^{\prime} \operatorname{Surf}_{2} \\
+\left\langle a^{\prime} x+b^{\prime} y \mid \mathrm{d} x \wedge \mathrm{d} y\right\rangle+\mathrm{d} w
\end{aligned}
$$

où le symbole ' désigne la dérivée par rapport à la variable $\rho$ et où $w$ est la 1-forme :

$$
w=c\langle y \mid \mathrm{d} x\rangle .
$$


L'analyse en type d'orbites est la suivante : il existe deux orbites singulières de type $S^{2}$, elles sont définies par les équations :

$$
S_{-}:\left\langle f_{m}(x) \mid y\right\rangle=-1 \text { et } S_{+}:\left\langle f_{m}(x) \mid y\right\rangle=+1,
$$

les autres orbites sont principales, elles ont comme type de stabilisateur $\mathbb{Z} / m \mathbb{Z}$.

Remarque 6.2. - La $\mathrm{SO}(3)$-variété $\mathcal{S}_{m}$ peut être obtenue aussi comme un fibré associé à la projection canonique $\pi: \mathrm{SO}(3) \rightarrow S^{2}$. En effet, on identifie $\mathrm{SO}(2)$ aux rotations de $S^{2}$ autour d'un axe, par exemple celui dirigé par le premier vecteur de base $(1,0,0)$; à tout entier $m \in \mathbb{N}$ on associe l'action suivante de $\mathrm{SO}(2)$ sur $S^{2}:(a, \xi) \mapsto a^{m}(\xi)$. On vérifie alors que $\mathcal{S}_{m}$ est isomorphe au produit fibré $\mathrm{SO}(3) \times_{\mathrm{SO}(2)} S^{2}$. Cela revient à considérer $S^{2}$ comme le compactifié de $\mathbb{R}^{2}$ et à prolonger la construction de $\mathcal{F}_{m}$.

\subsection{Le projectif $P^{2}(\mathbb{C})$.}

On considère l'espace projectif $P^{2}(\mathbb{C})$ muni de sa structure réelle; on l'obtient comme quotient de $S^{5}$ par $U(1)$, la sphère $S^{5}$, plongée dans $\mathbb{R}^{3} \times \mathbb{R}^{3}$, possède une action différentiable de $\mathrm{SO}(3)$; cette action passe au quotient et définit une action naturelle de $\mathrm{SO}(3)$ sur $P^{2}(\mathbb{C})$. Notons $(x, y)$ un point de $S^{5} \subset \mathbb{R}^{3} \times \mathbb{R}^{3}$, l'espace des orbites $P^{2}(\mathbb{C}) / \mathrm{SO}(3)$ est réalisé comme l'intervalle $[0,1]$ grâce à la projection :

$$
(x, y) \mapsto \rho=1-4\|x \wedge y\|^{2} .
$$

Toute application équivariante $L \in \mathcal{E} \mathrm{q}\left(P^{2}(\mathbb{C}), \mathbb{R}^{3}\right)$ s'écrit, relevée sur $S^{5}$ :

$$
L(x, y)=a(\rho) x \wedge y,
$$

où $a$ est une fonction différentiable réelle définie sur l'intervalle $[0,1]$. La 2-forme invariante $\sigma$ ayant $L$ comme moment s'écrit explictement avec quelques abus d'écriture :

$$
\sigma=\frac{1}{2}\left[\mathrm{~d}(a \omega)-a^{\prime} p^{*}\left(\operatorname{Surf}_{\mathbb{R}^{2}}\right)\right],
$$

où $p$ est la projection de $P^{2}(\mathbb{C})$ sur $\mathbb{R}^{2}$ définie par :

$$
p:(x, y) \mapsto\left(\|x\|^{2}-\|y\|^{2}, 2-\|x \wedge y\|^{2}\right) .
$$

L'analyse en type d'orbites est ici un peu plus délicate, il existe deux orbites singulières $\mathcal{O}_{\wedge}$ et $\mathcal{O}_{\perp}$ images des sous-variétés d'équations, respectivement :

$$
x \wedge y=0 \quad \text { et } \quad\|x \wedge y\|^{2}=\frac{1}{4} .
$$

TOME $119-1991-\mathrm{N}^{\circ} 3$ 
L'orbite $\mathcal{O}_{\wedge}$ est du type $P^{2}(\mathbb{R})$ et $\mathcal{O}_{\perp}$ est du type $S^{2}$. Le projectif $P^{2}(\mathbb{C})$ est obtenu par recollement des voisinages tubulaires de chaque orbites isomorphes respectivement à $T P^{2}(\mathbb{R})$ et $\mathcal{F}_{2}$.

\section{Classification des $\mathrm{SO}(3)$-variétés symplectiques de dimension 4}

Dans ce chapitre nous classons les $\mathrm{SO}(3)$-variétés symplectiques de dimension 4, nous montrons d'abord que leur type topologique est un de ceux décrits précédemment (chapitre 6), nous donnons ensuite pour chaque type topologique les structures symplectiques classées à difféomorphisme équivariant près. Plus précisément nous introduisons une relation de pré-ordre sur les structures symplectiques dont nous classons uniquement les éléments maximaux, nous montrons que le seul invariant de ces structures maximales est la classe de cohomologie de la forme symplectique.

\subsection{Type topologique.}

Le type topologique des $\mathrm{SO}(3)$-variétés symplectiques de dimension 4 est donnée par le théorème suivant où les notations $\mathcal{L}_{m}, \mathcal{F}_{m}, \mathcal{S}_{m}$ sont définies au chapitre 6 :

Théorème. - Les $\mathrm{SO}(3)$-variétés symplectiques de dimension 4 sont d'un des types différentiables suivants:

1) $S^{2} \times X$ où $\mathrm{SO}(3)$ agit naturellement sur $S^{2}$ et trivialement sur $X$; $X$ est symplectique et s'identifie à l'espace des orbites.

2) $\mathcal{L}_{m} ;$ toutes les orbites sont principales de type $\mathrm{SO}(3) /(\mathbb{Z} / m \mathbb{Z})$ et l'espace des orbites est la droite réelle.

3) $\mathcal{F}_{m}$; ce sont les fibrés de fibre $\mathbb{R}^{2}$ sur $S^{2}$ de classe de Chern $2 m$. La section nulle est l'orbite singulière de type $S^{2}$, toutes les autres orbites sont principales de type $\mathrm{SO}(3) /(\mathbb{Z} / m \mathbb{Z})$, l'espace des orbites est la demidroite fermée.

4) $T P^{2}(\mathbb{R})$; la section nulle est l'orbite singulière de type $P^{2}(\mathbb{R})$, les autres orbites sont principales de type $\mathrm{SO}(3) /(\mathbb{Z} / 2 \mathbb{Z})$, l'espace des orbites est la demi-droite fermée.

5) $\mathcal{S}_{m}$; c'est différentiablement le produit $S^{2} \times S^{2}$ mais avec une action de $\mathrm{SO}(3)$ indexée par $m$. L'action de $\mathrm{SO}(3)$ a 2 orbites singulières de types $S^{2}$, les autres orbites sont principales de type $\mathrm{SO}(3) /(\mathbb{Z} / m \mathbb{Z})$, l'espace des orbites est le segment fermé.

6) $P^{2}(\mathbb{C})$; l'action de $\mathrm{SO}(3)$ est induite par l'action naturelle de $\mathrm{SU}(3)$; elle a deux orbites singulières, l'une du type $S^{2}$ et l'autre du 
type $P^{2}(\mathbb{R})$; les autres orbites sont principales de type $\mathrm{SO}(3) /(\mathbb{Z} / 2 \mathbb{Z})$, l'espace des orbites est le segment fermé.

La lettre $m$ désigne un entier strictement positif quelconque. On remarque que les seules $\mathrm{SO}(3)$-variétés symplectiques de dimension 4 compactes sont différentiablement $S^{2} \times S^{2}$ et $P^{2}(\mathbb{C})$ (hormis le cas trivial $\left.S^{2} \times X\right)$.

Démonstration. - Soit $V$ une $\mathrm{SO}(3)$-variété symplectique. Nous savons que le type de stabilisateur principal est $\mathrm{SO}(2)$ ou bien un groupe cyclique $\mathbb{Z} / m \mathbb{Z}$ (THÉORÈme 3.1 ). Le premier cas a été traité au $\S 4$, il ne reste donc que le cas du stabilisateur cyclique, étant donné la dimension $\operatorname{de} V: \operatorname{dim} V=4$, l'espace des orbites est une variété à bord ou sans bord de dimension 1, c'est-à-dire d'un des types topologique :

$$
V / \mathrm{SO}(3) \simeq S^{1}, \quad \mathbb{R}, \quad[0, \infty[\quad \text { ou } \quad[-1,+1] .
$$

Les points du bord de $V / \mathrm{SO}(3)$ sont les images des orbites singulières.

1) Le premier cas est interdit parce que le petit hamiltonien canonique $h$ défini sur $V / \mathrm{SO}(3)$ n'a pas de points critiques (Proposition 5.4).

2) Dans le cas $V / \mathrm{SO}(3)=\mathbb{R}$ toutes les orbites sont principales, la variété $V$ est isomorphe au produit $[\mathrm{SO}(3) /(\mathbb{Z} / m \mathbb{Z})] \times \mathbb{R}$, c'est-à-dire à la $\mathrm{SO}(3)$-variété que nous avons notée $\mathcal{L}_{m}$ (voir $\S 6.1$ ) et dont nous avons décrit les structures symplectiques invariantes.

3) Dans le cas $V / \operatorname{SO}(3) \simeq[0, \infty[$ il n'y a qu'une orbite singulière du type $S^{2}$ ou $P^{2}(\mathbb{R})$ en vertu de la Proposition 5.4, $V$ est alors obtenu par le théorème de la tranche; c'est un fibré associé à la projection $\mathrm{SO}(3) \rightarrow S^{2}$ ou $\mathrm{SO}(3) \rightarrow P^{2}(\mathbb{R})$ de fibre $\mathbb{R}^{2}$. Dans le premier cas le stabilisateur singulier $\mathrm{SO}(2) \subset \mathrm{SO}(3)$ agit orthogonalement sur $\mathbb{R}^{2}$ et transitivement sur la sphère unité; la seule action de ce type est donnée par $(a, x) \mapsto a^{m} x$ où $(a, x) \in \mathrm{SO}(2) \times \mathbb{R}^{2}$. On obtient ainsi les fibrés $\mathcal{F}_{m}$ décrits au $\S 6.2$. Dans le deuxième cas, le stabilisateur singulier est $\mathrm{O}(2) \subset \mathrm{SO}(3)$ qui n'a qu'une action orthogonale sur $\mathbb{R}^{2}$ qui soit transitive sur la sphère unité, c'est l'action standard; le fibré obtenu est alors isomorphe au fibré tangent à $P^{2}(\mathbb{R})$ dont les structures symplectique invariantes ont été décrites au $§ 6.3$.

4) Dans le cas $V / \mathrm{SO}(3) \simeq[-1,+1], V$ est compacte, il y a deux orbites singulières corespondant aux points du bord. Chaque orbite possède un voisinage tubulaire qui est encore une $\mathrm{SO}(3)$-variété symplectique, mais cette fois du type précédent, c'est-à-dire : $\mathcal{F}_{m}$ ou $T P^{2}(\mathbb{R})$. Pour décrire la topologie de $V$ on peut choisir deux voisinages tubulaires fermés $\mathcal{U}_{-}$et $\mathcal{U}_{+}$, le bord de chacun de ces voisinages est une orbite du type $\mathrm{SO}(3) /(\mathbb{Z} / m \mathbb{Z})$ si le stabilisateur principal est lui-même du type $\mathbb{Z} / m \mathbb{Z}$. La variété $V$

TOME $119-1991-\mathrm{N}^{\circ} 3$ 
s'obtient alors par recollement des voisinages $\mathcal{U}_{-}$et $\mathcal{U}_{+}$le long du bord par un difféomorphisme équivariant, le résultat topologique ne dépend pas du difféomorphisme. Nous voyons que les seuls candidats au recollement sont :

$$
\mathcal{F}_{m} \cup \mathcal{F}_{m} \quad \text { ou } \quad \mathcal{F}_{2} \cup T P^{2}(\mathbb{R}) \quad \text { ou } \quad T P^{2}(\mathbb{R}) \cup T P^{2}(\mathbb{R}) .
$$

L'analyse en type d'orbites du premier cas donne : deux orbites singulières de type $S^{2}$ et les stabilisateurs principaux du type $\mathbb{Z} / m \mathbb{Z}$; on reconnaît la $\mathrm{SO}(3)$-variété $\mathcal{S}_{m}$ décrite au $\S 6.4$. L'analyse en type d'orbites du deuxième cas donne : une orbite singulière de type $S^{2}$, une autre du type $P^{2}(\mathbb{R})$ et les stabilisateurs principaux du type $\mathbb{Z} / 2 \mathbb{Z}$; on reconnait la $\mathrm{SO}(3)$ variété $P^{2}(\mathbb{C})$ décrite au $\S 6.5$. Le troisième cas est interdit, en effet le petit hamiltonien canonique s'annulerait nécessairement sur les deux orbites singulières qui sont chacunes du type $P^{2}(\mathbb{R})$ (donc lagrangiennes), ceci impliquerait l'existence d'un point critique à l'intérieur du segment $[-1,+1]$ ce qui est interdit par la Proposition 5.4. Il ne reste donc que les variétés annoncées.

\section{2. Équivalence et ordre.}

Il est naturel de classer les $\mathrm{SO}(3)$-variétés symplectiques à difféomorphisme équivariant près, deux $\mathrm{SO}(3)$-variétés symplectiques $(V, \omega)$ et $\left(V^{\prime}, \omega^{\prime}\right)$ sont équivalentes s'il existe un difféomorphisme équivariant $\varphi$ de $V$ dans $V^{\prime}$ :

$$
\forall A \in \mathrm{SO}(3), \forall x \in V, \quad \varphi(A x)=A \varphi(x)
$$

tel que :

$$
\varphi^{*}\left(\omega^{\prime}\right)=\omega .
$$

On s'aperçoit rapidement que la classification à difféomorphisme équivariant près est encore trop large : tout ouvert $\mathrm{SO}(3)$-invariant $\Omega$ de la variété symplectique $(V, \omega)$ munie de la structure symplectique induite est encore une $\mathrm{SO}(3)$-variété symplectique. Pour éliminer ces cas inutiles nous introduisons la relation suivante :

DÉFINITION 7.2. - Etant données deux $\mathrm{SO}(3)$-variétés symplectiques $(V, \omega)$ et $\left(V^{\prime}, \omega^{\prime}\right)$, nous dirons que $\omega$ est antérieure $\grave{a} \omega^{\prime}$, ou que $\omega^{\prime}$ prolonge $\omega$, et nous noterons : $\omega \preceq \omega^{\prime} s^{\prime}$ 'il existe un plongement équivariant $\varphi$ de $V$ sur un ouvert de $V^{\prime}$ tel que : $\varphi^{*}\left(\omega^{\prime}\right)=\omega$. Nous dirons que $\omega$ est strictement antérieure $\grave{a} \omega^{\prime}$, ou que $\omega^{\prime}$ prolonge strictement $\omega$, et nous noterons : $\omega \prec \omega^{\prime}$ si $\omega \preceq \omega^{\prime}$ et $\varphi(V) \neq V^{\prime}$. 
Remarque 7.3. - Compte tenu des notations précédentes, il est évident que si $\omega$ est antérieure à $\omega^{\prime}$ et $V$ est compacte alors $V^{\prime}$ est aussi compacte et $\varphi$ est un difféomorphisme symplectique de $V$ sur $V^{\prime}$, les $\mathrm{SO}(3)$-variété symplectiques sont alors équivalentes. Le critère d'antériorité que nous venons d'introduire n'a d'intérêt que si $V$ n'est pas compacte.

Proposition 7.4. - Tout plongement symplectique équivariant d'une $\mathrm{SO}(3)$-variété symplectique de dimension 4 à stabilisateur principal cyclique dans elle-même est surjectif.

Démonstration. - Soit $(V, \omega)$ une $\mathrm{SO}(3)$-variété symplectique de dimension 4 à stabilisateur principal cyclique; on suppose qu'il existe un plongement symplectique équivariant strict $\varphi$ de $V$ dans $V: \varphi^{*} \omega=\omega$ et $\varphi(V) \neq V$. On déduit immédiatement que $V$ n'est pas compacte et donc que $V / \mathrm{SO}(3) \simeq]-\infty,+\infty[$ ou encore $V / \mathrm{SO}(3) \simeq[0, \infty[$. Le plongement $\varphi$ se projette sur un plongement $f$ de l'espace des orbites dans lui-même et on a immédiatement $h \circ f=h$ où $h$ est le petit hamiltonien canonique de $\omega$ (formule (7)). Par hypothèse $f(V / \mathrm{SO}(3)) \neq V / \mathrm{SO}(3)$, on peut donc supposer par exemple que $\lim _{x \rightarrow \infty} f(x)=a \in \mathbb{R}$, on a alors $\lim _{x \rightarrow \infty} h(x)=h(a)$ ce qui est impossible car $h$ est strictement monotone.

Par application immédiate de la proposition précédente (7.4) on déduit le corollaire suivant :

Corollaire 7.5. - Définie sur les classes de $\mathrm{SO}(3)$-variétés symplectiques de dimension 4 à stabilisateur principal cyclique, la relation d'antériorité est une relation d'ordre partiel.

Nous dirons donc qu'une $\mathrm{SO}(3)$-variété symplectique $(V, \omega)$ est maximale si elle n'est strictement antérieure à aucune autre. La proposition suivante nous conduira à ne classer, à difféomorphisme équivariant près, en dimension 4 que les $\mathrm{SO}(3)$-variétés symplectique maximales.

Proposition 7.6. - Toute $\mathrm{SO}(3)$-variété symplectique de dimension 4 à stabilisateur principal cyclique est antérieure à une $\mathrm{SO}(3)$-variété symplectique maximale.

Démonstration. - Considérons une famille totalement ordonnées $\left(V_{i}, \omega_{i}\right)_{i \in I}$ de $\mathrm{SO}(3)$-variétés symplectiques non-compactes, les petits hamiltoniens canoniques sont notés $h_{i}, i \in I$. Soit $\omega_{i} \preceq \omega_{j}$, et choisissons $\varphi$ un plongement équivariant de $V_{i}$ dans $V_{j}$ tel que $\varphi^{*}\left(\omega_{j}\right)=\omega_{i}$. Le plongement $\varphi$ se projette naturellement en un plongement $f$ de l'espace des orbites $V_{i} / \mathrm{SO}(3)$ dans $V_{j} / \mathrm{SO}(3)$ tel que $h_{j} \circ f=h_{i}$. La famille d'intervalles $U_{i}=h_{i}\left(V_{i} / \mathrm{SO}(3)\right) \subset[0, \infty[, i \in I$, est donc totalement ordonnée 
pour l'inclusion, nous pouvons choisir une sous-famille dénombrable d'intervalles $\left(U_{\alpha}\right)_{\alpha \in A}$ où $A \subset I$ telle que $\cup_{\alpha \in A} U_{\alpha}=\cup_{i \in I} U_{i}$. Il est alors possible, pour tout couple d'indices $(\alpha, \beta) \in A \times A$ tel que $\omega_{\alpha} \preceq \omega_{\beta}$, de choisir une plongement symplectique équivariant $\varphi_{\alpha}^{\beta}: V_{\alpha} \rightarrow V_{\beta}$ et de construire ainsi une famille inductive de $\mathrm{SO}(3)$-variétés symplectiques dont la limite est un majorant de $\left(V_{i}, \omega_{i}\right)_{i \in I}$. On applique alors le lemme de Zorn.

La relation d'antériorité entre $\mathrm{SO}(3)$-variétés symplectiques à stabilisateur principal cyclique est schématisée sur la Figure 1. Ce graphe est obtenu en comparant la stucture en type d'orbites. On voit que pour $i \neq 2$ les seules possibilités de prolongement sont $\mathcal{L}_{i} \rightarrow \mathcal{F}_{i} \rightarrow \mathcal{S}_{i}$. Pour $i=2$, outre le prolongement précédent $\mathcal{L}_{2} \rightarrow \mathcal{F}_{2} \rightarrow \mathcal{S}_{2}$, on a : $\mathcal{L}_{2} \rightarrow T P(2, \mathbb{R}) \rightarrow P(2, \mathbb{C})$ et $\mathcal{L}_{2} \rightarrow \mathcal{F}_{2} \rightarrow P(2, \mathbb{C})$.

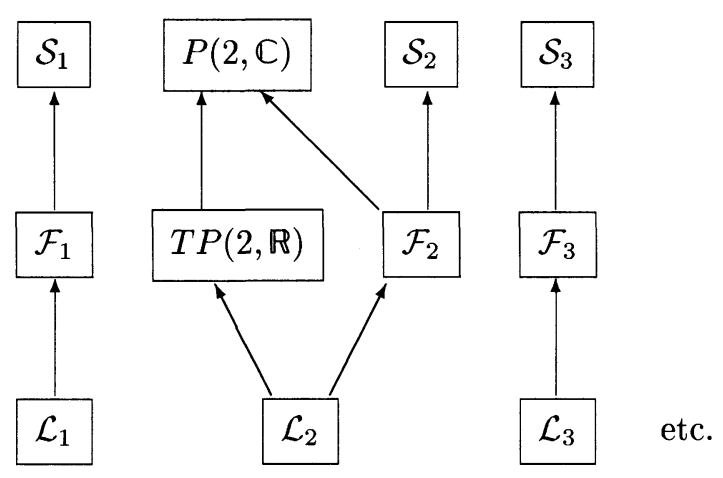

Figure 1. Antériorité des $\mathrm{SO}(3)$-variétés

\subsection{Classification.}

Les $\mathrm{SO}(3)$-variétés de dimension 4 décrites dans la liste du THÉoRÈmE 7.1 rentrent dans le cadre des théorèmes, aujourd'hui classiques, sur la structure fonctionnelle des espaces d'orbites (chapitres VI.5 et VI.6 de [Bre72]). De ces théorèmes on déduit que tout difféomorphisme $f$ de $V / \mathrm{SO}(3)$ se relève en un difféomorphisme équivariant $\varphi$ de $V$. Ceci permet d'établir la proposition :

Proposition 7.7. - Soit $V$ une $\mathrm{SO}(3)$-variété de dimension 4 à stabilisateur principal cyclique. Si $\omega_{1}$ et $\omega_{2}$ sont deux formes symplectiques invariantes sur $V$ telles que $h_{1}(V / \mathrm{SO}(3))=h_{2}(V / \mathrm{SO}(3))$, il existe une difféomorphisme équivariant $\phi$ de $V$ tel que $\omega_{2}=\phi^{*} \omega_{1}$. 
Démonstration. - Soit $(V, \omega)$ une $\mathrm{SO}(3)$-variété symplectique de dimension 4 à stabilisateur principal cyclique. Soient $L$ son moment équivariant et $h$ son petit hamiltonien canonique. Soit $\varphi$ un difféomorphisme équivariant de $V$ et $f$ sa projection sur la variété des orbites $V / \mathrm{SO}(3)$. Soit $\omega^{*}$ l'image réciproque de $\sigma$ par $\varphi$ de moment équivariant $L^{*}$ et de petit hamiltonien canonique $h^{*}$. On a évidemment les égalités suivantes :

$$
L^{*}=L \circ \varphi \quad h^{*}=h \circ f .
$$

Donc, si $h_{1}(V / \mathrm{SO}(3))=h_{2}(V / \mathrm{SO}(3))$ la permutation unique $f$ de $V / \mathrm{SO}(3)$ définie par $h_{2}=h_{1} \circ f$ est un difféomorphisme (propriété des $h_{i}$ ), elle se relève alors en un difféomorphisme équivariant $\varphi$ de $V$. On est ramené au cas de deux moments équivariants $L_{1}$ et $L_{2}$ qui ont même petit hamiltonien canonique $h$.

Si l'action de $\mathrm{SO}(3)$ est principales, les moments ne s'annulent nulle part et il n'est pas difficile de construire une isogénie de $V$ qui envoie $L_{1}$ sur $L_{2}$. Plus délicat lorsque les moments s'annulent, nécessairement sur une seule orbite singulière $\mathcal{O}$; on peut montrer que l'isogénie construite sur la réunion des orbites principales se prolonge sur l'orbite singulière par une analyse du germe de l'isogénie au voisinage de l'orbite singulière; dans son dernier travail, Thomas Delzant [Del90] utilise une méthode puissante de fonction génératrice ce qui simplifie notablement la démonstration.

En d'autres termes, les seuls invariants de l'action du groupe des difféomorphismes équivariants sur les formes symplectiques invariantes de $V$ (répondant aux hypothèses de la proposition précédente) sont les valeurs extrèmes du petit hamiltonien canonique $h$. Il nous reste maintenant à donner la liste des $\mathrm{SO}(3)$-variétés symplectiques de dimension 4 maximales, elle s'obtient en fonction des valeurs extrèmes du petit hamiltonien canonique. La proposition suivante est donnée sans démonstration, le lecteur intéressé se reportera à [Igl84]; rappelons que le cas du stabilisateur principal de type $\mathrm{SO}(2)$ a été traité en toute dimension au chapitre 4; le symbole $\simeq$ désigne l'équivalence des $\mathrm{SO}(3)$-variétés différentiables.

Proposition 7.8. - Soit $(V, \omega)$ une $\mathrm{SO}(3)$-variété symplectique de dimension 4 à stabilisateur principal cyclique, soit $L$ son moment équivariant, alors :

1) L'action de $\mathrm{SO}(3)$ est libre :

- $V \simeq \mathcal{L}_{1}=\mathrm{SO}(3) \times \mathbb{R}$ et la structure symplectique $\omega$ se prolonge $\grave{a}$ $T S^{2}$ ou encore à $\mathcal{S}_{1}=S^{2} \times S^{2}$.

- $V \simeq T S^{2} ; \omega$ est maximale si et seulement si son moment $L$ s'écrit (à équivalence près) $L(x, v)=s x+x \wedge v$. Toute structure invariante de volume finie est prolongeable à $\mathcal{S}_{1}=S^{2} \times S^{2}$.

TOME $119-1991-\mathrm{N}^{\circ} 3$ 
- $V \simeq \mathcal{S}_{1}=S^{2} \times S^{2}, L$ est donné à équivalence près par $L(x, y)=$ $s_{1} x+s_{2} y$ avec et $0<s_{1}<s_{2}$.

2) Le type de stabilisateur principal est $\mathbb{Z} / 2 \mathbb{Z}$ :

- $V \simeq \mathcal{L}_{2}=[\mathrm{SO}(3) /(\mathbb{Z} / 2 \mathbb{Z})] \times \mathbb{R}$ et la structure symplectique $\omega$ se prolonge à $T P^{2}(\mathbb{R})$ ou encore à $P^{2}(\mathbb{C})$.

- $V \simeq \mathcal{F}_{2}$ (fibré $\mathbb{R}^{2}$ de classe de Chern 4 sur $\left.S^{2}\right)$; l'orbite singulière est la section nulle; $\omega$ est maximale si et seulement si le moment équivariant s'écrit $L(x, v)=s e^{E} x$ où $E=\frac{1}{2}\|v\|^{2}$ et $s>0$. Toute structure invariante de volume finie est prolongeable à $\mathcal{S}_{2}$ ou à $P^{2}(\mathbb{C})$.

- $V \simeq T P^{2}(\mathbb{R}) ;$ l'orbite singulière est la section nulle : $\omega$ est maximale si et seulement si elle est équivalente à la structure canonique de cotangent.

- $V \simeq P^{2}(\mathbb{C})$; il y a deux orbites singulières l'une du type $S^{2}$ l'autre du type $P^{2}(\mathbb{R})$, w est équivalente à la forme symplectique canonique d'orbite coadjointe de $\mathrm{SU}(3)$ (ou encore définie par la connexion de Hopf).

- $V \simeq \mathcal{S}_{2}$, voir plus loin.

3) Le type de stabilisateur principal est $\mathbb{Z} / m \mathbb{Z}(m>2)$ :

- $V$ est équivalent à $\mathcal{F}_{m}$ privé de la section nulle c'est-à-dire à $\mathcal{L}_{m}$; $\omega$ est maximale si et seulement si son moment équivariant s'écrit à équivalence près $L(x, v)=E x$ avec $E=\frac{1}{2}\|v\|^{2}$.

- $V \simeq \mathcal{F}_{m} ;$ l'orbite singulière est la section nulle; $\omega$ est maximale si et seulement si son moment équivariant s'écrit à équivalence près d'une des deux façons suivantes: $L(x, v)=s e^{E} x$ ou $L(x, v)=s e^{-E} x$ avec $E=\frac{1}{2}\|v\|^{2}$.

- $V \simeq \mathcal{S}_{m}(m \geq 2)$; les orbites singulières sont du type $S^{2}$; le moment équivariant de $\omega$ s'écrit à équivalence près : $L(x, y)=\left(s_{1}^{2}+2 s_{1} s_{2} \rho+s_{2}^{2}\right)^{1 / 2} x$ avec $0<s_{1}<s_{2}$ et $\rho=\left\langle f_{m}(x) \mid y\right\rangle$ (voir définition de $f_{m}$ formule (27))

Remarque 7.9. - Le seul invariant des structures symplectiques maximales est la classe de cohomologie de $\omega$. 


\section{BIBLIOGRAPHIE}

[Aud88] Audin (M.). - Hamiltoniens périodiques sur les variétés symplectiques compactes de dimension 4. - Prétirage 369P/P-204, Institut de Recherche mathématique Avancé de Strasbourg, 1988.

[Aud89] Audin (M.). - Opérations hamiltoniennes des tores sur les variétés symplectiques. - Polycopié, Institut de Recherche mathématique Avancé de Strasbourg, 1989.

[Bre72] Bredon (G.E.). - Introduction to compact transformation groups. Academic Press, New-York, $197^{2}$.

[Del88] Delzant (T.). - Hamiltoniens périodiques et images convexes de l'application moment, Bulletin de la SMF, t. 116, 1988.

[Del90] Delzant (T.). - Classifications des actions hamiltoniennes complètement intégrables des groupes de rang 2, Annals of Global Analysis and Geometry, t. 8 (1), 1990, p. 87-112.

[Ig184] Iglesias (P.). - Classification des $\mathrm{SO}(3)$-variétés symplectiques de dimension 4. - Prétirage CPT-84/PE.1673, CPT-CNRS, Luminy, Marseille (France), 1984.

[MW74] J. Marsden and A. Weinstein. - On reduction of symplectic manifold, Rep. Math. Phys., t. 5, 1974, p. 421.

[Pal60] Palais (P.). - The classification of G-spaces, Mem. Amer. Math. Soc, t. 36, 1960 .

[Poé76] Poénaru (V.). - Singularité $\mathcal{C}^{\infty}$ en présence de symétrie. — Lecture Notes in Mathematics, 510, 1976.

[Sou70] Souriau (J.M.). - Structure des systèmes dynamiques. - Dunod, Paris, 1970.

[Wol72] Wolf (J.). - Spaces of constant curvature. - Berkeley, Californie, 1972. 